\title{
First complete mitogenomes of Diamesinae, Orthocladiinae, Prodiamesinae, Tanypodinae (Diptera: Chironomidae) and their implication in phylogenetics
}

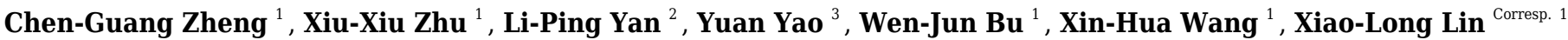 \\ ${ }^{1}$ College of Life Sciences, Nankai University, Tianjin, China \\ 2 School of Ecology and Nature Conservation, Beijing Forestry University, Beijing, China \\ 3 College of Life Sciences, Tianjin Normal University, Tianjin, China \\ Corresponding Author: Xiao-Long Lin \\ Email address: lin880224@gmail.com
}

Background. The mitochondrial genome (mitogenome) has been extensively used for phylogenetic and evolutionary analysis in Diptera, but the study of mitogenome is still scarce in the family Chironomidae.

Methods. Here, the first complete mitochondrial genomes of four Chironomid species representing Diamesinae, Orthocladiinae, Prodiamesinae and Tanypodinae are presented. Coupled with published mitogenomes of two, a comparative mitochondrial genomic analysis between six subfamilies of Chironomidae was carried out.

Results. Mitogenomes of Chironomidae are conserved in structure, each contains 37 typical genes and a control region, and all genes arrange the same gene order as the ancestral insect mitogenome.

Nucleotide composition is highly biased, the control region displayed the highest A+T content. All protein coding genes are under purifying selection, and the ATP8 evolves at the fastest rate. In addition, the phylogenetic analysis covering six subfamilies within Chironomidae was conducted. The monophyly of Chironomidae is strongly supported. However, the topology of six subfamilies based on mitogenomes in this study is inconsistent with previous morphological and molecular studies. This may be due to the high mutation rate of the mitochondrial genetic markers within Chironomidae. Our results indicate that mitogenomes showed poor signals in phylogenetic reconstructions at the subfamily level of Chironomidae. 


\section{First complete mitogenomes of Diamesinae,}

2 Orthocladiinae, Prodiamesinae, Tanypodinae (Diptera:

Chen-Guang Zheng ${ }^{1}$, Xiu-Xiu Zhu ${ }^{1}$, Li-Ping Yan ${ }^{2}$, Yuan Yao ${ }^{3}$, Wen-Jun Bu${ }^{1}$, Xin-Hua Wang ${ }^{1}$,

7 Xiao-Long Lin ${ }^{1, *}$

8

${ }^{1}$ College of Life Sciences, Nankai University, Tianjin, 300071, P. R. China

${ }^{2}$ School of Ecology and Nature Conservation, Beijing Forestry University, Beijing, 100083, P.

R. China

${ }^{3}$ College of Life Sciences, Tianjin Normal University, Tianjin, 300387, P. R. China

Corresponding Author:

Xiao-Long $\operatorname{Lin}^{1}$

94 Weijin Road, Tianjin, 300071, P. R. China

Email address: lin880224@gmail.com

\section{Abstract}

Background. The mitochondrial genome (mitogenome) has been extensively used for phylogenetic and evolutionary analysis in Diptera, but the study of mitogenome is still scarce for the family Chironomidae.

Methods. Complete mitochondrial genomes of four chironomid species representing the Diamesinae, Orthocladiinae, Prodiamesinae and Tanypodinae are presented. Coupled with the published mitogenomes of two additional subfamilies, a comparative mitochondrial genomic analysis of six subfamilies of Chironomidae was carried out.

Results. Mitogenomes of Chironomidae are conserved in structure, each contains the typical 37 genes and a control region. All genes are arranged with the same order as the ancestral insect mitogenome. Nucleotide composition is highly biased with the control region displaying the highest $\mathrm{A}+\mathrm{T}$ content. All protein coding genes are under purifying selection, and ATP8 evolves at the fastest rate. The monophyly of Chironomidae is strongly supported, however, relationships between the six subfamilies based on mitogenomes in this study is inconsistent with previous morphological and molecular studies. This may be due to the high mutation rate of mitochondrial genetic markers within Chironomidae. Our results indicate that mitogenomes have poor signal for phylogenetic reconstructions at the subfamily level of Chironomidae.

\section{Introduction}


72

73

74

75

76

77

78

The typical mitochondrial genome (mitogenome) of insects is a double-strand circular molecule ranging from $14 \mathrm{~kb}$ to $20 \mathrm{~kb}$ in size, which encodes 37 genes (13 protein-coding genes, two ribosomal RNA genes, and 22 transfer RNA genes) and a control region (Boore 1999; Cameron 2014; Wolstenholme 1992). Due to its small genome size, maternal inheritance, low sequence recombination, and fast evolutionary rates (Brown et al. 1979; Curole \& Kocher 1999), the mitogenome is considered as powerful marker for phylogenetic and evolutionary analysis (Condamine et al. 2018; Jacobsen et al. 2012; Stokkan et al. 2018; Tang et al. 2019b). Due to high-throughput sequencing technology, an increasing number of complete mitogenomes have been sequenced among the Diptera, covering most families (Kang et al. 2016; Li et al. 2020; Miao et al. 2020; Ramakodi et al. 2015; Tang et al. 2019a). Mitogenomes have been widely used for mitochondrial structure comparison and phylogenetic analysis at different taxonomic level of the Diptera (Chen et al. 2018; de Oliveira Aragão et al. 2019; Miao et al. 2020; Yan et al. 2019; Zhang et al. 2016; Zhang et al. 2019b). However, complete mitogenomes are still scarce for the family Chironomidae, which limits our understanding of their mitochondrial structure and phylogenetic pattern. In addition, it is still unknown whether mitogenomes can effectively resolve phylogenetic relationships at the subfamily level within Chironomidae.

The dipteran family Chironomidae is a diverse aquatic insect group, and are important bioindicators for freshwater ecosystem monitoring. Within Chironomidae, several phylogenetic studies have been conducted based on morphological characters or combining genetic markers to reconstruct the evolutionary history of subfamilies (Cranston et al. 2012; Sæther 1977), but no one has attempted to use mitogenomes. Prior to this study, only five mitogenomes of Chironomidae were available (Beckenbach 2012; Deviatiiarov et al. 2017; Kim et al. 2016; Park et al. 2020; Zhang et al. 2019a), representing species from three subfamilies: Chironominae, Podonominae, and Prodiamesinae. However, comparative analysis of mitogenome structure, base composition, substitution and evolutionary rates among subfamilies has not been carried out. In addition, the monophyly of Chironomidae has not been supported by a recent study using mitogenomes of Culicomorpha (Zhang et al. 2019b).

In the present study, we provide complete mitogenomes for four species representing the subfamilies Diamesinae, Orthocladiinae, Prodiamesinae, and Tanypodinae. Along with the published mitogenomes of subfamilies Chironominae and Podonominae, the first comparative analysis of the genome structure, base composition, substitution and evolutionary rates among six chironomid subfamilies is presented. In addition, a phylogenomic analysis covering six chironomid subfamilies was carried out.

\section{Materials \& Methods}

\section{Taxon sampling}

Complete mitogenomes of six chironomid species (Appendix S1), representing six subfamilies, were analyzed in this study, with two ceratopogonid species used as outgroups. The mitogenomes of four non-biting midge species, Potthastia sp. (Diamesinae), Rheocricotopus villiculus (Orthocladiinae), Prodiamesa olivacea (Prodiamesinae) and Clinotanypus yani 
79 (Tanypodinae) are documented for the first time. The mitogenomes of Chironomus tepperi

80 (Chironominae) and Parochlus steinenii (Podonominae) were retrieved from GenBank

81 (Beckenbach 2012; Kim et al. 2016). The mitogenome of Propsilocerus akamusi (MN566452)

82 (Zhang et al. 2019a) was excluded from the present study because it is incomplete and lacks

83 annotation. In addition, two species of Ceratopogonidae (Culicoides arakawae and Forcipomyia

84 makanensis) (Jiang et al. 2019; Matsumoto et al. 2009) were selected as outgroups for

85 phylogenetic analyses since Ceratopogonidae was strongly supported as the sister group of

86 Chironomidae in previous studies (Kutty et al. 2018). Detailed taxon sampling information is

87 listed in Table 1. The vouchers of the newly sequenced species are deposited at the college of

88 Life Sciences, Nankai University, Tianjin, China.

89 DNA extraction, sequencing and assembling

90 For the newly sequenced species, total genomic DNA was extracted from the body, (except

91 abdomen and genitalia) using a General AllGen Kit (Qiagen, Germany). The entire mitogenome

92 of each species were sequenced using the Illumina NovaSeq 6000 platform with an insert size of

93 350-bp and a paired-end 150-bp sequencing strategy by the Allwegene Company and Novogene

94 Co., Ltd. at Beijing, China. About $2 \mathrm{~Gb}$ clean data were obtained from each library after

95 trimming using Trimmomatic (Bolger et al. 2014).

96 To ensure the accuracy of the mitogenome sequences, three frequently used assembly methods

97 were applied to each sample. A de novo assembly was performed using IDBA-UD (Peng et al.

98 2012) with minimum and maximum $\mathrm{k}$ values of 40 and $120 \mathrm{bp}$, respectively. Two reference

99 based assemblies were performed using Geneious 2020.2.1 (Kearse et al. 2012) with default

100 setting and MITObim 1.9 (Hahn et al. 2013). The mitogenome sequences obtained by the three

101 methods were aligned, manually compared, and finally compiled into a single sequence in

102 Geneious 2020.2.1 (Kearse et al. 2012).

103 Genome annotation, composition and substitution rate

104 Genome annotation was conducted as previously described in Zheng et al. (2020). Specifically,

105 the transfer RNA (tRNA) genes and their secondary structures were detected by MITOS2

106 webserver (http://mitos2.bioinf.uni-leipzig.de/index.py) (Bernt et al. 2013) with invertebrate

107 mitochondrial genetic code. The ribosomal RNA (rRNA) genes were predicted by alignment

108 with homologous regions of mitogenome from closely related species. Protein coding genes

109 (PCGs) were initially annotated using the Open Reading Frame Finder (ORF Finder) as

110 implemented at the NCBI website (https://www.ncbi.nlm.nih.gov/orffinder/) and then compared

111 with published mitogenomes of insects using the program BLAST

112 (http://blast.ncbi.nlm.nih.gov/Blast.cgi). Newly sequenced mitogenomes were submitted to

113 GenBank (accession numbers: MW373523-MW373526).

114 CGView Server V 1.0 (Grant \& Stothard 2008) was used to draw mitogenome maps. Codon

115 usage of PCGs and nucleotide composition were calculated in MEGA X (Kumar et al. 2018).

116 The bias of the nucleotide composition was measured according to the formulas: AT-skew $=(\mathrm{A}-$

$117 \mathrm{~T}) /(\mathrm{A}+\mathrm{T})$ and $\mathrm{GC}$-skew $=(\mathrm{G}-\mathrm{C}) /(\mathrm{G}+\mathrm{C})$. Rates of non-synonymous substitution rate $(\mathrm{Ka})$ and

118 synonymous substitution rate (Ks) were calculated in DnaSP 6.12.03 (Rozas et al. 2017).

119 Phylogenetic analyses

Peer] reviewing PDF | (2020:12:56227:2:0:NEW 24 Mar 2021) 
120 Phylogenetic analyses were conducted using the sequences of 13 PCGs and two rRNAs. The 121 PCGs were aligned based on amino acid sequences using Muscle implemented in MEGA X 122 (Kumar et al., 2018). The rRNAs were aligned using MAFFT 7.402 (Katoh \& Standley 2013) 123 with algorithm G-INS-i strategy. Alignments of individual genes were then concatenated using 124 SequenceMatrix v1.7.8 (Vaidya et al. 2011) to generate five datasets: PCG123 (all three codon

125

126

127

128

129

130

131

132

133

134

135

136

137

138

139

140

141

142

143

144

145

146

147

148

149

150

151

152

153

154

155

156

157

158

159

positions of the 13 PCGs), PCG123R (all three codon positions of the 13 PCGs and two rRNAs), PCG12 (the first and second codon positions of the 13 PCGs), PCG12R (the first and second codon positions of the 13 PCGs and two rRNAs), and AA (amino acid sequences of the 13 PCGs). To test substitution saturation, transition and transversion rates were evaluated by DAMBE 5.6.14 (Xia 2013). The program PartitionFinder 2.0 (Lanfear et al. 2017) was used to infer the best substitution model (Table S1). The analysis of Bayesian inference (BI) and maximum likelihood (ML) were conducted for each dataset. The BI analyses were performed under the program MrBayes 3.2.7a (Ronquist et al. 2012) with partitioned models (Table S1). Two simultaneous Markov chain Monte Carlo (MCMC) runs of 10,000,000 generations were conducted, trees were sampled every 1000 generations with a burn-in of $25 \%$. The program Tracer 1.7 (Rambaut et al. 2018) was used to assess the convergence of runs. The ML analyses were conducted using the program RAxML 8.0.12 (Stamatakis 2014) under the substitution model GTR+GAMMA+I. The nodal support values were calculated with 1000 bootstrap replicates.

\section{Results}

\section{Mitogenome organization and composition}

The complete mitogenomes of Chironomus tepperi, Potthastia sp., Rheocricotopus villiculus, Parochlus steinenii, Prodiamesa olivacea, and Clinotanypus yani are 15,652, 15,913, 15,985, 16,803, 16,190, and 16,247 bp in size, respectively (Fig. 1; Appendix S2). They are circular molecules, each containing 37 typical mitochondrial genes (13 PCGs, two rRNAs, and 22 tRNAs) and one control region. Among these genes, four PCGs (ND1, ND4, ND4L, and ND5), eight tRNAs (trnC, trnF, trnH, trnL (UAG), trnP, trnQ, trnV, and trnY), and two rRNAs (12S rRNAs and 16S rRNAs) are encoded by the minority strand (N strand), while the other 23 genes are located in the majority strand (J strand). ATP8-ATP6 and ND4L-ND4 overlap by seven nucleotides (ATGATAA and ATGTTAA, respectively) in all six Chironomidae species.

Nucleotide composition (Table 2) of the six Chironomidae species is similar, with a high A+T bias $(72.4 \%-76.8 \%)$, the control region has the highest $\mathrm{A}+\mathrm{T}$ content while the first and the second codon positions of PCGs have the lowest A+T content. All six Chironomidae species exhibited negative AT-skew and GC-skew. All three codon positions of PCGs had negative ATskew, the GC-skew of the first codon position was positive, while the 2 nd and the $3 \mathrm{rd}$ codon position were negative. Some gene regions exhibited different nucleotide skew among the six Chironomidae species. For example, in 12S rRNA, the AT-skew of Chironomus tepperi and Clinotanypus yani are -0.01 and 0.00 respectively, while the AT-skew are positive $(0.01-0.05)$ in the remaining four species.

Peer] reviewing PDF | (2020:12:56227:2:0:NEW 24 Mar 2021) 
160

161

162

163

164

165

166

167

168

169

170

171

172

173

174

175

176

177

178

179

180

181

182

183

184

185

186

187

188

189

190

191

192

193

194

195

196

197

198

199

\section{Protein coding genes}

Among Chironomidae species, most PCGs initiate with the standard start codon ATN. The start codon of COI was TTG in Chironomus tepperi, Potthastia sp., Rheocricotopus villiculus and Prodiamesa olivacea. The start codon of ND5 in Chironomus tepperi, Potthastia sp., Rheocricotopus villiculus, Prodiamesa olivacea and Clinotanypus yani was GTG. ND1 started with TTG in Potthastia sp., Rheocricotopus villiculus, Parochlus steinenii, Prodiamesa olivacea, and Clinotanypus yani. Most PCGs have complete termination codons (TAA or TAG), however, COII in Parochlus steinenii and Clinotanypus yani has an incomplete termination codon (T-).

Total codon number (except the termination codons) in Chironomus tepperi, Potthastia sp., Rheocricotopus villiculus, Parochlus steinenii, Prodiamesa olivacea, and Clinotanypus yani were $3730,3743,3726,3729,3729$, and 3709, respectively. The most frequent codon families are Ile, Leu2, and Phe $(>300)$, while the least used codon family is Cys $(<50)$ in all six Chironomidae species (Fig. 2). The relative synonymous codon usage (RSCU) patterns among the six Chironomidae species are similar. The RSCU values are showed in Fig. 3. All synonymous codons of 20 amino acids are present. The most frequent used codons are NNU and NNA for each amino acid (Fig. 3).

The $\mathrm{Ka} / \mathrm{Ks}$ value $(\omega)$ was used to test for signatures of natural selection (Cheng et al. 2018; $\mathrm{Hu}$ $\&$ Banzhaf 2008). The $\omega$ value of all PCGs are less than 0.6. Among the 13 PCGs, ATP8 has the largest $\omega$ value, indicating that ATP8 evolves at the fastest rate. The animal DNA barcoding gene COI has the lowest $\omega$ value (Fig. 4).

tRNAs, rRNAs and control region

The typical set of 22 tRNA genes were identified in the mitogenomes of all six Chironomidae species, ranging from 63 to $72 \mathrm{bp}$ in length. The tRNA genes exhibit high A+T content $(73.2 \%-$ $79.5 \%$ ), positive AT-skew, and negative GC-skew (Table 2). All the predicted tRNAs can be folded into the typical clover-leaf secondary structure except $\operatorname{trnS}$ (GCU), which lacks the dihydrouridine (DHU) arm. The non-Watson-crick base pair G-U is common in tRNA genes from all Chironomidae species (Fig. S1-S6).

Both 12S and 16S rRNA genes exhibit similar position and size across the Chironomidae mitogenomes. The $\mathrm{A}+\mathrm{T}$ content of $12 \mathrm{~S}$ and $16 \mathrm{~S}$ rRNA genes ranges from $76.4 \%$ to $82.6 \%$ and $80.1 \%$ to $84.4 \%$, respectively. Both genes exhibit positive AT-skew and negative GC-skew in all Chironomidae species except Chironomus tepperi: the AT-skew of 12S rRNA and 16S rRNA in Chironomus tepperi is -0.01 and 0.00 , respectively (Table 2 ).

The control regions of Chironomus tepperi, Potthastia sp., Rheocricotopus villiculus, Parochlus steinenii, Prodiamesa olivacea, and Clinotanypus yani are 500, 911, 832, 1783, 1079, and $1095 \mathrm{bp}$ in size, respectively (Appendix S2). All are A+T rich (85.5\%-93.7\%), much higher than the whole mitogenomes $(72.4 \%-77.3 \%)$.

\section{Saturation test and phylogenetic analyses}

Saturation tests were performed for the four nucleotide datasets. Each dataset was free of saturation (Fig. S7). In general, phylogenetic trees support the monophyly of the Chironomidae across different datasets in ML and BI analyses (Fig. 5, PP =1, BS =100). Within 
200 Chironomidae, four topologies were inferred from five datasets: i) Orthocladiinae +

201 (Chironominae $+(($ Diamesinae + Prodiamesinae $)+($ Podonominae + Tanypodinae $)))$ was

202 inferred from the PCG123 and PCGR datasets (Fig. 5a,5b); ii) Orthocladiinae + (Chironominae +

203 (Diamesinae $+($ Prodiamesinae $+($ Podonominae + Tanypodinae $)))$ was inferred from the PCG12

204 dataset (Fig. 5c); iii) (Orthocladiinae + Chironominae $)+($ Diamesinae $+($ Prodiamesinae +

205 (Podonominae + Tanypodinae))) was inferred from the PCG12R dataset (Fig. 5d); iv)

206 Chironominae $+($ Orthocladiinae + (Prodiamesinae $+($ Diamesinae $+($ Podonominae +

207 Tanypodinae)))) was inferred from the AA dataset (Fig. 5e). The topology inferred from the AA

208 had the strongest nodal support. Based on five different datasets, Podonominae is sister to

209 Tanypodinae with strong support in both BI ( $P P \geq 0.98)$ and, ML $(B S=100)$ reconstructions,

210 which makes the sister to (Diamesinae + Prodiamesinae) with strong support $(\mathrm{PP}=1, \mathrm{BS} \geq 91)$

211 at the "tip" position. The remaining subfamilies Chironominae and Orthocladiinae are sister to

212 above four subfamilies.

213

214 Discussion

\section{Mitogenome features}

216 The entire mitogenome length of the six Chironomidae species differs considerably $(15,652$

217 16,803), mainly due to the variation in control region size. All Chironomidae mitogenomes

218 contain 37 typical genes and a control region, the order and arrangement of these genes are

219 completely accordant with the ancestral insect gene arrangement (Clary \& Wolstenholme 1985).

220 The whole mitogenome of Chironomidae has high A+T content and similar AT/GC-skew,

221 consistent with the similar base composition biases of insect mitochondrial DNA (Wei et al.

222 2010). This type of nucleotide bias may be related to the asymmetric mutation processes during

223 replication (Hassanin et al. 2005).

224 Among the Chironomidae mitogenomes, most PCGs have complete termination codons, while

225 the COII gene in Parochlus steinenii and Clinotanypus yani has an incomplete termination codon

226 (T-) that probably completed by post-transcriptional polyadenylation (Ojala et al. 1981). The

227 patterns of codon usage among the Chironomidae mitogenomes are nearly the same. The most

228 frequent used codons were NNU and NNA for each amino acid, reflecting the AT bias of

229 nucleotide composition. For most amino acids, the most frequently used codon is not the anti-

230 codon that strictly correspond to tRNA. The low $\omega$ value for each PCG indicates that they are all

231 under strong purifying selection. The animal DNA barcoding gene COI has the lowest

232 evolutionary rate, which is consistent with the results observed from other insect groups ( $\mathrm{Li}$ et al.

233 2020; Yang et al. 2013; Zhang \& Ye 2017).

234 All six Chironomidae mitogenomes contain the 22 typical tRNA genes, and secondary

235 structure across species is similar. Unlike other tRNA genes, $\operatorname{trnS}$ (GCU) lacks the

236 dihydrouridine (DHU) arm. This could be commonly found in published insect mitogenomes ( $\mathrm{Li}$

237 et al. 2012; Lu et al. 2020; Zhang et al. 2018). The A+T contents of 12S rRNA, 16S rRNA, and

238 control region are much higher than that in the whole genome in Chironomidae mitogenomes,

239 indicating a strong $\mathrm{A}+\mathrm{T}$ bias in these regions. 


\section{Phylogenetic analyses}

241 In this study, we applied a variety of strategies to explore the phylogenetic relationships of six 242 subfamilies within the Chironomidae, and confirmed the monophyly of Chironomidae (Fig. 5).

243 However, the topology of subfamilies based on mitogenomes in this study is inconsistent with 244 previous morphological and molecular studies (Cranston et al. 2012; Sæther 1977; Sæther 2000).

245 The present morphological phylogenetics of Chironomidae (Sæther 2000) is composed 11 246 subfamilies, including $(((($ Chironominae + Orthocladiinae $)+$ Prodiamesinae $)+$ Diamesinae $)+$ 247 Buchonomyiinae + Chienomyiinae $)+(($ Usambaromyiinae + Tanypodinae $)+$ Podonominae + 248 Aphroteniinae)) + Telmatogetoninae. The present molecular phylogenetic system of 249 Chironomidae (Cranston et al. 2012) is composed nine subfamilies, including ((()((Chironominae $250+($ Orthocladiinae + Prodiamesinae $))+$ Diamesinae $)+$ Telmatogetoninae $)+$ Tanypodinae $)+$ 251 Podonominae) + Aphroteniinae) + Buchonomyiinae. Nevertheless, Podonominae and 252 Tanypodinae are ancestral taxa based on both traditional morphological and molecular 253 phylogenies. However, they appear at the "tip" position of mitogenomic phylogenetic tree.

254 Moreover, the "tip" taxa Chironominae and Orthocladiinae appear at the "root" position of the 255 mitogenomic phylogenetic tree. This erroneous phylogenetic reconstruction may be a result of 256 long branch attraction (LBA) (Siddall \& Whiting 1999). Due to the high mutation rate of the 257 mitochondrial genetic markers within Chironomidae, some studies (Ekrem \& Willassen 2004;

258 Ekrem et al. 2010) have reported that mitochondrial markers (e.g. COI, COII) are not suitable for 259 phylogenetic relationship reconstruction. Here, our mt data reveal different evolutionary history 260 of six subfamilies, which is contradictory with traditional morphology-based systematics.

261 Therefore, we assume that mitogenomes has poor signal for phylogenetic reconstructions at 262 subfamily level in the Chironomidae.

263

264 Conclusions

265 In this study, we sequenced four complete mitogenomes representing four subfamilies of 266 Chironomidae by whole genome sequencing technologies and did the first comparative analysis 267 of mitogenome base composition and evolutionary history in Chironomidae. The study shows 268 that mitogenomes of Chironomidae are conserved in structure, gene order and nucleotide 269 composition. Our results revealed that mitogenomes have poor phylogenetic signals for 270 subfamily level relationships in Chironomidae.

271

\section{Acknowledgements}

273 A big thank to Dr. Lidong Mo for his help on the manuscript improvement. We also thank Dr. 274 Andrey Krasheninnikov and another two anonymous reviewers for their constructive comments. 275

\section{References}

Beckenbach AT. 2012. Mitochondrial genome sequences of Nematocera (lower Diptera): evidence of rearrangement following a complete genome duplication in a winter crane fly. Genome Biology and Evolution 4:89-101. 10.1093/gbe/evr131 
280

281

282

283

284

285

286

287

288

289

290

291

292

293

294

295

296

297

298

299

300

301

302

303

304

305

306

307

308

309

310

311

312

313

314

315

316

317

318

319

320

321

322

323

324

325

Bernt M, Donath A, Jühling F, Externbrink F, Florentz C, Fritzsch G, Pütz J, Middendorf M, and Stadler PF. 2013. MITOS: improved de novo metazoan mitochondrial genome annotation. Molecular Phylogenetics and Evolution 69:313-319. 10.1016/j.ympev.2012.08.023

Bolger AM, Lohse M, and Usadel B. 2014. Trimmomatic: a flexible trimmer for Illumina sequence data. Bioinformatics 30:2114-2120. 10.1093/bioinformatics/btu170

Boore JL. 1999. Animal mitochondrial genomes. Nucleic Acids Research 27:1767-1780. 10.1093/nar/27.8.1767

Brown WM, George M, and Wilson AC. 1979. Rapid evolution of animal mitochondrial DNA. Proceedings of the National Academy of Sciences 76:1967-1971. 10.1073/pnas.76.4.1967

Cameron SL. 2014. Insect mitochondrial genomics: implications for evolution and phylogeny. Annual Review of Entomology 59:95-117. 10.1146/annurev-ento-011613-162007

Chen J-Y, Chang Y-W, Zheng S-Z, Lu M-X, and Du Y-Z. 2018. Comparative analysis of the Liriomyza chinensis mitochondrial genome with other Agromyzids reveals conserved genome features. Scientific Reports 8:1-10. 10.1038/s41598-018-27213-7

Cheng YC, Chen MY, Wang JF, Liang AP, and Lin CP. 2018. Some mitochondrial genes perform better for damselfly phylogenetics: species - and population - level analyses of four complete mitogenomes of Euphaea sibling species. Systematic Entomology 43:702-715. 10.1111/syen.12299

Clary DO, and Wolstenholme DR. 1985. The mitochondrial DNA molecule of Drosophila yakuba: nucleotide sequence, gene organization, and genetic code. Journal of Molecular Evolution 22:252-271.

Condamine FL, Nabholz B, Clamens A-L, Dupuis JR, and Sperling FA. 2018. Mitochondrial phylogenomics, the origin of swallowtail butterflies, and the impact of the number of clocks in Bayesian molecular dating. Systematic Entomology 43:460-480. 10.1111/syen.12284

Cranston PS, Hardy NB, and Morse GE. 2012. A dated molecular phylogeny for the Chironomidae (Diptera). Systematic Entomology 37:172-188. 10.1111/j.1365-3113.2011.00603.x

Curole JP, and Kocher TD. 1999. Mitogenomics: digging deeper with complete mitochondrial genomes. Trends in Ecology \& Evolution 14:394-398. 10.1016/S0169-5347(99)01660-2

de Oliveira Aragão A, Neto JPN, Cruz ACR, Casseb SMM, Cardoso JF, da Silva SP, and Ishikawa EAY. 2019. Description and phylogeny of the mitochondrial genome of Sabethes chloropterus, Sabethes glaucodaemon and Sabethes belisarioi (Diptera: Culicidae). Genomics 111:607-611. 10.1016/j.ygeno.2018.03.016

Deviatiiarov R, Kikawada T, and Gusev O. 2017. The complete mitochondrial genome of an anhydrobiotic midge Polypedilum vanderplanki (Chironomidae, Diptera). Mitochondrial DNA Part A 28:218220. 10.3109/19401736.2015.1115849

Ekrem T, and Willassen E. 2004. Exploring Tanytarsini relationships (Diptera : Chironomidae) using mitochondrial COIl gene sequences. Insect Syst Evol 35:263-276. $10.1163 / 187631204788920248$

Ekrem T, Willassen E, and Stur E. 2010. Phylogenetic utility of five genes for dipteran phylogeny: A test case in the Chironomidae leads to generic synonymies. Molecular Phylogenetics and Evolution 57:561-571. 10.1016/j.ympev.2010.06.006

Grant JR, and Stothard P. 2008. The CGView Server: a comparative genomics tool for circular genomes. Nucleic Acids Research 36:181-184. 10.1093/nar/gkn179

Hahn C, Bachmann L, and Chevreux B. 2013. Reconstructing mitochondrial genomes directly from genomic next-generation sequencing reads - a baiting and iterative mapping approach. Nucleic Acids Research 41:e129-e129. 10.1093/nar/gkt371 
326

327

328

329

330

331

332

333

334

335

336

337

338

339

340

341

342

343

344

345

346

347

348

349

350

351

352

353

354

355

356

357

358

359

360

361

362

363

364

365

366

367

368

369

370

371

372
Hassanin A, Leger N, and Deutsch J. 2005. Evidence for multiple reversals of asymmetric mutational constraints during the evolution of the mitochondrial genome of Metazoa, and consequences for phylogenetic inferences. Systematic Biology 54:277-298. 10.1080/10635150590947843

Hu T, and Banzhaf W. 2008. Nonsynonymous to Synonymous Substitution Ratio ka/ks: Measurement for Rate of Evolution in Evolutionary Computation. International Conference on Parallel Problem Solving from Nature: Springer. p 448-457.

Jacobsen MW, Hansen MM, Orlando L, Bekkevold D, Bernatchez L, Willerslev E, and Gilbert MTP. 2012. Mitogenome sequencing reveals shallow evolutionary histories and recent divergence time between morphologically and ecologically distinct European whitefish (Coregonus spp.). Molecular Ecology 21:2727-2742. 10.1111/j.1365-294X.2012.05561.x

Jiang X, Han X, Liu Q, and Hou X. 2019. The mitochondrial genome of Forcipomyia makanensis (Insecta: Diptera: Ceratopogonidae). Mitochondrial DNA Part B 4:344-345. 10.1080/23802359.2018.1544048

Kang Z, Li X, and Yang D. 2016. The complete mitochondrial genome of Dixella sp. (Diptera: Nematocera, Dixidae). Mitochondrial DNA Part A 27:1528-1529. 10.3109/19401736.2014.953123

Katoh K, and Standley DM. 2013. MAFFT multiple sequence alignment software version 7: improvements in performance and usability. Molecular Biology and Evolution 30:772-780. $10.1093 / \mathrm{molbev} / \mathrm{mst010}$

Kearse M, Moir R, Wilson A, Stones-Havas S, Cheung M, Sturrock S, Buxton S, Cooper A, Markowitz S, and Duran C. 2012. Geneious Basic: an integrated and extendable desktop software platform for the organization and analysis of sequence data. Bioinformatics 28:1647-1649. 10.1093/bioinformatics/bts199

Kim S, Kim H, and Shin SC. 2016. Complete mitochondrial genome of the Antarctic midge Parochlus steinenii (Diptera: Chironomidae). Mitochondrial DNA Part A 27:3475-3476. 10.3109/19401736.2015.1066355

Kumar S, Stecher G, Li M, Knyaz C, and Tamura K. 2018. MEGA X: molecular evolutionary genetics analysis across computing platforms. Molecular Biology and Evolution 35:1547--1549. $10.1093 / \mathrm{molbev} / \mathrm{msy} 096$

Kutty SN, Wong WH, Meusemann K, Meier R, and Cranston PS. 2018. A phylogenomic analysis of Culicomorpha (Diptera) resolves the relationships among the eight constituent families. Systematic Entomology 43:434-446. 10.1111/syen.12285

Lanfear R, Frandsen PB, Wright AM, Senfeld T, and Calcott B. 2017. PartitionFinder 2: new methods for selecting partitioned models of evolution for molecular and morphological phylogenetic analyses. Molecular Biology and Evolution 34:772-773. 10.1093/molbev/msw260

Li H, Liu H, Shi A, Štys P, Zhou X, and Cai W. 2012. The complete mitochondrial genome and novel gene arrangement of the unique-headed bug Stenopirates sp. (Hemiptera: Enicocephalidae). PloS One 7:e29419. 10.1371/journal.pone.0029419

Li X-Y, Yan L-P, Pape T, Gao Y-Y, and Zhang D. 2020. Evolutionary insights into bot flies (Insecta: Diptera: Oestridae) from comparative analysis of the mitochondrial genomes. International Journal of Biological Macromolecules 149:371-380. 10.1016/j.ijbiomac.2020.01.249

Lu C, Huang X, and Deng J. 2020. The challenge of Coccidae (Hemiptera: Coccoidea) mitochondrial genomes: The case of Saissetia coffeae with novel truncated tRNAs and gene rearrangements. International Journal of Biological Macromolecules 158:854-864. 10.1016/j.ijbiomac.2020.04.257

Matsumoto Y, Yanase T, Tsuda T, and Noda H. 2009. Species - specific mitochondrial gene rearrangements in biting midges and vector species identification. Medical and Veterinary Entomology 23:47 - 55. 10.1111/j.1365-2915.2008.00789.x

Peer] reviewing PDF | (2020:12:56227:2:0:NEW 24 Mar 2021) 
373

374

375

376

377

378

379

380

381

382

383

384

385

386

387

388

389

390

391

392

393

394

395

396

397

398

399

400

401

402

403

404

405

406

407

408

409

410

411

412

413

414

415

416

417

418

419

420
Miao X, Huang J, Menzel F, Wang Q, Wei Q, Lin X-L, and Wu H. 2020. Five mitochondrial genomes of black fungus gnats (Sciaridae) and their phylogenetic implications. International Journal of Biological Macromolecules 150:200-205. 10.1016/j.ijbiomac.2020.01.271

Ojala D, Montoya J, and Attardi G. 1981. tRNA punctuation model of RNA processing in human mitochondria. Nature 290:470-474.

Park K, Jo H, Choi B, and Kwak I-S. 2020. Complete mitochondrial genome of Stictochironomus akizukii (Tokunaga) (Chironomidae, Diptera) assembled from next-generation sequencing data. Mitochondrial DNA Part B 5:2310-2311. 10.1080/23802359.2020.1750320

Peng Y, Leung HC, Yiu S-M, and Chin FY. 2012. IDBA-UD: a de novo assembler for single-cell and metagenomic sequencing data with highly uneven depth. Bioinformatics 28:1420-1428. 10.1093/bioinformatics/bts174

Ramakodi MP, Singh B, Wells JD, Guerrero F, and Ray DA. 2015. A 454 sequencing approach to dipteran mitochondrial genome research. Genomics 105:53-60. 10.1016/j.ygeno.2014.10.014

Rambaut A, Drummond AJ, Xie D, Baele G, and Suchard MA. 2018. Posterior summarization in Bayesian phylogenetics using Tracer 1.7. Systematic Biology 67:901-904. 10.1093/sysbio/syy032

Ronquist F, Teslenko M, van der Mark P, Ayres DL, Darling A, Höhna S, Larget B, Liu L, Suchard MA, and Huelsenbeck JP. 2012. MrBayes 3.2: efficient Bayesian phylogenetic inference and model choice across a large model space. Systematic Biology 61:539-542. 10.1093/sysbio/sys029

Rozas J, Ferrer-Mata A, Sánchez-DelBarrio JC, Guirao-Rico S, Librado P, Ramos-Onsins SE, and SánchezGracia A. 2017. DnaSP 6: DNA sequence polymorphism analysis of large data sets. Molecular Biology and Evolution 34:3299-3302. 10.1093/ molbev/msx248

Sæther OA. 1977. Female genitalia in Chironomidae and other Nematocera: morphology, phylogenies, keys. Bulletin of the Fisheries Research Board of Canada 197:1-209.

Sæther OA. 2000. Phylogeny of the subfamilies of Chironomidae (Diptera). Systematic Entomology 25:393-403. 10.1046/j.1365-3113.2000.00111.x

Siddall ME, and Whiting MF. 1999. Long - branch abstractions. Cladistics 15:9 - 24.

Stamatakis A. 2014. RAxML version 8: a tool for phylogenetic analysis and post-analysis of large phylogenies. Bioinformatics 30:1312-1313. 10.1093/bioinformatics/btu033

Stokkan M, Jurado-Rivera JA, Oromí P, Juan C, Jaume D, and Pons J. 2018. Species delimitation and mitogenome phylogenetics in the subterranean genus Pseudoniphargus (Crustacea: Amphipoda). Molecular Phylogenetics and Evolution 127:988-999. doi.org/10.1016/j.ympev.2018.07.002

Tang L, Yan L, Gao Y, and Zhang D. 2019a. First report of mitochondrial genome from the subfamily Bengaliinae (Diptera: Calliphoridae). Mitochondrial DNA Part B 4:1560-1561. 10.1080/23802359.2019.1601037

Tang P, Zhu J-c, Zheng B-y, Wei S-j, Sharkey M, Chen X-x, and Vogler AP. 2019b. Mitochondrial phylogenomics of the Hymenoptera. Molecular Phylogenetics and Evolution 131:8-18. 10.1016/j.ympev.2018.10.040

Vaidya G, Lohman DJ, and Meier R. 2011. SequenceMatrix: concatenation software for the fast assembly of multi-gene datasets with character set and codon information. Cladistics 27:171-180. 10.1111/j.1096-0031.2010.00329.x

Wei S-J, Shi M, Chen X-X, Sharkey MJ, van Achterberg C, Ye G-Y, and He J-H. 2010. New views on strand asymmetry in insect mitochondrial genomes. PloS One 5:e12708. 10.1371/journal.pone.0012708

Wolstenholme DR. 1992. Animal mitochondrial DNA: structure and evolution. International Review of Cytology 141:173-216. 10.1016/S0074-7696(08)62066-5

Xia XH. 2013. DAMBE5: a comprehensive software package for data analysis in molecular biology and evolution. Molecular Biology and Evolution 30:1720-1728. 10.1093/molbev/mst064

Peer) reviewing PDF | (2020:12:56227:2:0:NEW 24 Mar 2021) 
421

422

423

424

425

426

427

428

429

430

431

432

433

434

435

436

437

438

439

440

441

442

443

444

445

446

447

448

449

450

451

452

453

454

455

456

457

458

Yan L, Pape T, Elgar MA, Gao Y, and Zhang D. 2019. Evolutionary history of stomach bot flies in the light of mitogenomics. Systematic Entomology 44:797-809. 10.1111/syen.12356

Yang W, Yu W, and Du Y. 2013. The complete mitochondrial genome of the sycamore lace bug Corythucha ciliata (Hemiptera: Tingidae). Gene 532:27-40. 10.1016/j.gene.2013.08.087

Zhang D, Yan L, Zhang M, Chu H, Cao J, Li K, Hu D, and Pape T. 2016. Phylogenetic inference of calyptrates, with the first mitogenomes for Gasterophilinae (Diptera: Oestridae) and Paramacronychiinae (Diptera: Sarcophagidae). International Journal of Biological Sciences 12:489-504. 10.7150/ijbs.12148

Zhang H-L, and Ye F. 2017. Comparative mitogenomic analyses of praying mantises (Dictyoptera, Mantodea): origin and evolution of unusual intergenic gaps. International Journal of Biological Sciences 13:367-382. 10.7150/ijbs.17035

Zhang L-P, Cai Y-Y, Yu D-N, Storey KB, and Zhang J-Y. 2018. Gene characteristics of the complete mitochondrial genomes of Paratoxodera polyacantha and Toxodera hauseri (Mantodea: Toxoderidae). PeerJ 6:e4595. 10.7717/peerj.4595

Zhang Q, Xu W, Peng K, Zou L, Li Y, Chen Y, Cai Y, and Gong Z. 2019a. The complete mitochondrial genome of Propsilocerus akamusi (Diptera, Chironomidae). Mitochondrial DNA Part B 4:39833984. 10.1080/23802359.2019.1688703

Zhang X, Kang Z, Ding S, Wang Y, Borkent C, Saigusa T, and Yang D. 2019b. Mitochondrial genomes provide insights into the phylogeny of Culicomorpha (Insecta: Diptera). International Journal of Molecular Sciences 20:747. 10.3390/ijms20030747

Zheng C, Ye Z, Zhu X, Zhang H, Dong X, Chen P, and Bu W. 2020. Integrative taxonomy uncovers hidden species diversity in the rheophilic genus Potamometra (Hemiptera: Gerridae). Zoologica Scripta 49:174-186. 10.1111/zsc.12401

\section{Figure captions}

Figure 1. Mitogenome maps of Chironomus tepperi (a), Potthastia sp. (b), Rheocricotopus villiculus (c), Rheocricotopus villiculus (d), Prodiamesa olivacea (e), Clinotanypus yani (f).

The names of PCGs and rRNAs are indicated by standard abbreviations, while names of tRNAs are represented by a single letter abbreviation. The first circle shows the gene map and arrows indicate the orientation of gene transcription. Blue arrows refer to PCGs, purple arrows refer to rRNAs, red arrows refer to tRNAs and grey arrow refers to control region. The second circle shows the GC content, which is plotted as the deviation from the average GC content of the entire sequence. The third circle shows the GC-skew, which is plotted as the deviation from the average GC-skew of the entire sequence. The innermost circle shows the sequence length.

Figure 2. Patterns of codon usage of the six mitogenomes of six chironomid subfamilies. The $\mathrm{X}$-axis shows the codon families and the $\mathrm{Y}$-axis shows the total codons.

\section{Figure 3. The relative synonymous codon usage (RSCU) in the six mitogenomes of six}

chironomid subfamilies. The $\mathrm{X}$-axis shows the codons and the $\mathrm{Y}$-axis shows RSCU values. 
459 Figure 4. Evolution rate of each PCG of the six mitogenomes of six chironomid subfamilies

460 mitogenomes. Ka refers to non-synonymous substitution rate, Ks refers to synonymous

461 substitution rate, $\mathrm{Ka} / \mathrm{Ks}$ refers to evolution rate of each $\mathrm{PCG}$.

462 Figure 5. Phylogenetic relationships of six subfamilies within Chironomidae inferred from

463 mitogenomes. (a) Topology obtained based on PCG123; (b) Topology obtained based on

464 PCG123R; (c) Topology obtained based on PCG12; (d) Topology obtained based on PCG12R;

465 (e) Topology obtained based on AA. Numbers at the nodes are BI posterior probabilities (left)

466 and ML bootstrap values (right).

467

468 Supporting Information

469 Table S1. The best model for each partition of the five datasets.

470 Figure S1. Secondary structure of 22 tRNAs in Chironomus tepperi.

471 Figure S2. Secondary structure of 22 tRNAs in Potthastia sp..

472 Figure S3. Secondary structure of 22 tRNAs in Rheocricotopus villiculus.

473 Figure S4. Secondary structure of 22 tRNAs in Parochlus steinenii.

474 Figure S5. Secondary structure of 22 tRNAs in Prodiamesa olivacea.

475 Figure S6. Secondary structure of 22 tRNAs in Clinotanypus yani.

476 Figure S7. Substitution patterns of the PCG123 (a), PCG123R (b), PCG12 (c), and PCG12R

477 (d) datasets. The graphs represent the increase in GTR distance.

478 Appendix S1. Mitogenome sequences of the six chironomid subfamilies and two outgroups.

479 Appendix S2. Organization of mitochondrial genomes of the six chironomid subfamilies. 


\section{Figure 1}

Mitogenome maps of Chironomus tepperi (a), Potthastia sp. (b), Rheocricotopus villiculus (c), Rheocricotopus villiculus (d), Prodiamesa olivacea (e), Clinotanypus yani (f).

The names of PCGs and rRNAs are indicated by standard abbreviations, while names of tRNAs are represented by a single letter abbreviation. The first circle shows the gene map and arrows indicate the orientation of gene transcription. Blue arrows refer to PCGs, purple arrows refer to rRNAs, red arrows refer to tRNAs and grey arrow refers to control region. The second circle shows the GC content, which is plotted as the deviation from the average GC content of the entire sequence. The third circle shows the GC-skew, which is plotted as the deviation from the average GC-skew of the entire sequence. The innermost circle shows the sequence length. 

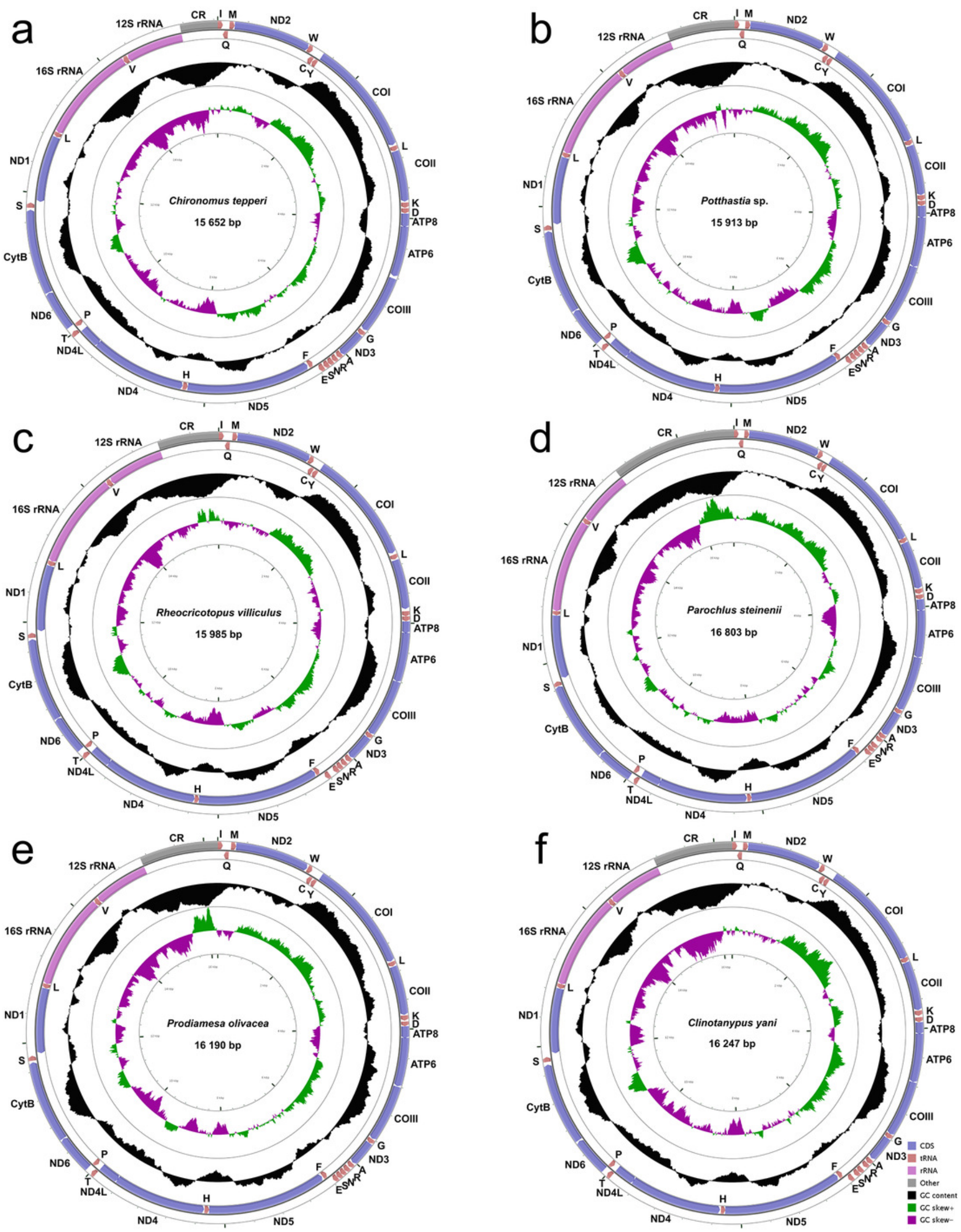


\section{Figure 2}

Patterns of codon usage of the six mitogenomes of six chironomid subfamilies.

The $\mathrm{X}$-axis shows the codon families and the $\mathrm{Y}$-axis shows the total codons.

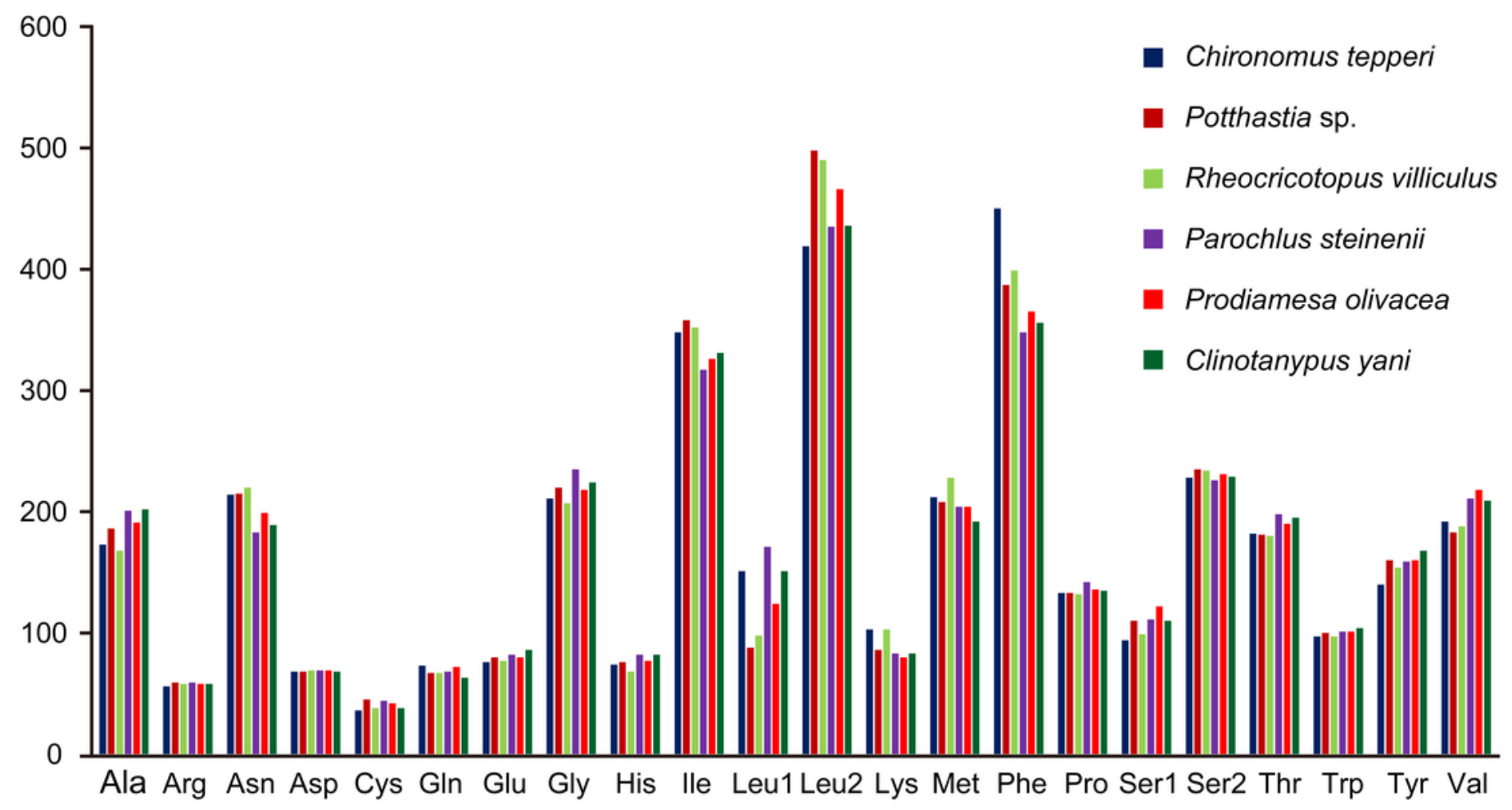


Figure 3

The relative synonymous codon usage (RSCU) in the six mitogenomes of six chironomid subfamilies.

The X-axis shows the codons and the Y-axis shows RSCU values. 

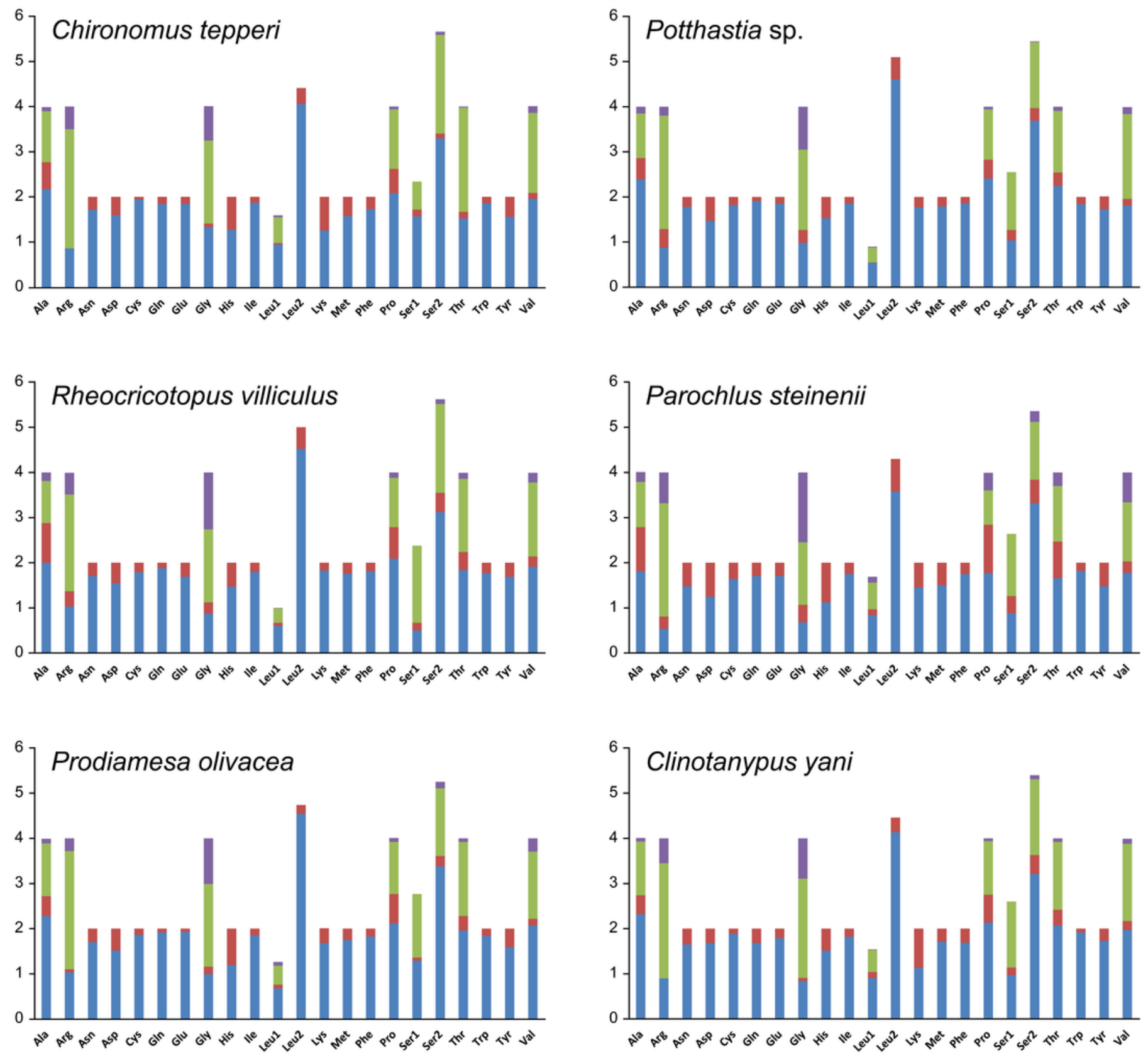

Ala Arg Asn Asp Cys Gln Glu Gly His lle Leu1 Leu2 Lys Met Phe Pro Ser1 Ser2 Thr Trp Tyr Val

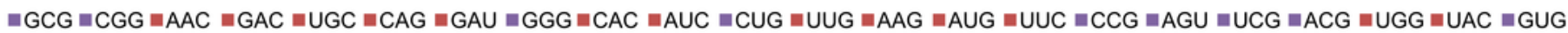

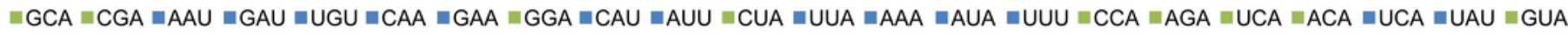
$\because G C C \backsim C G C$ $\because G G C$ $=C U C$ $\square \mathrm{CCC} \equiv \mathrm{AGC}=\mathrm{UCC} \equiv \mathrm{ACC}$ =GUC घGU $=\mathrm{CGU}$ -GGU $\square \mathrm{CUU}$ $\approx \mathrm{CCU} \approx \mathrm{AGU}=\mathrm{UCU} \approx \mathrm{ACU}$ aUU 
Figure 4

Evolution rate of each PCG of the six mitogenomes of six chironomid subfamilies mitogenomes.

Ka refers to non-synonymous substitution rate, Ks refers to synonymous substitution rate, $\mathrm{Ka} / \mathrm{Ks}$ refers to evolution rate of each PCG.

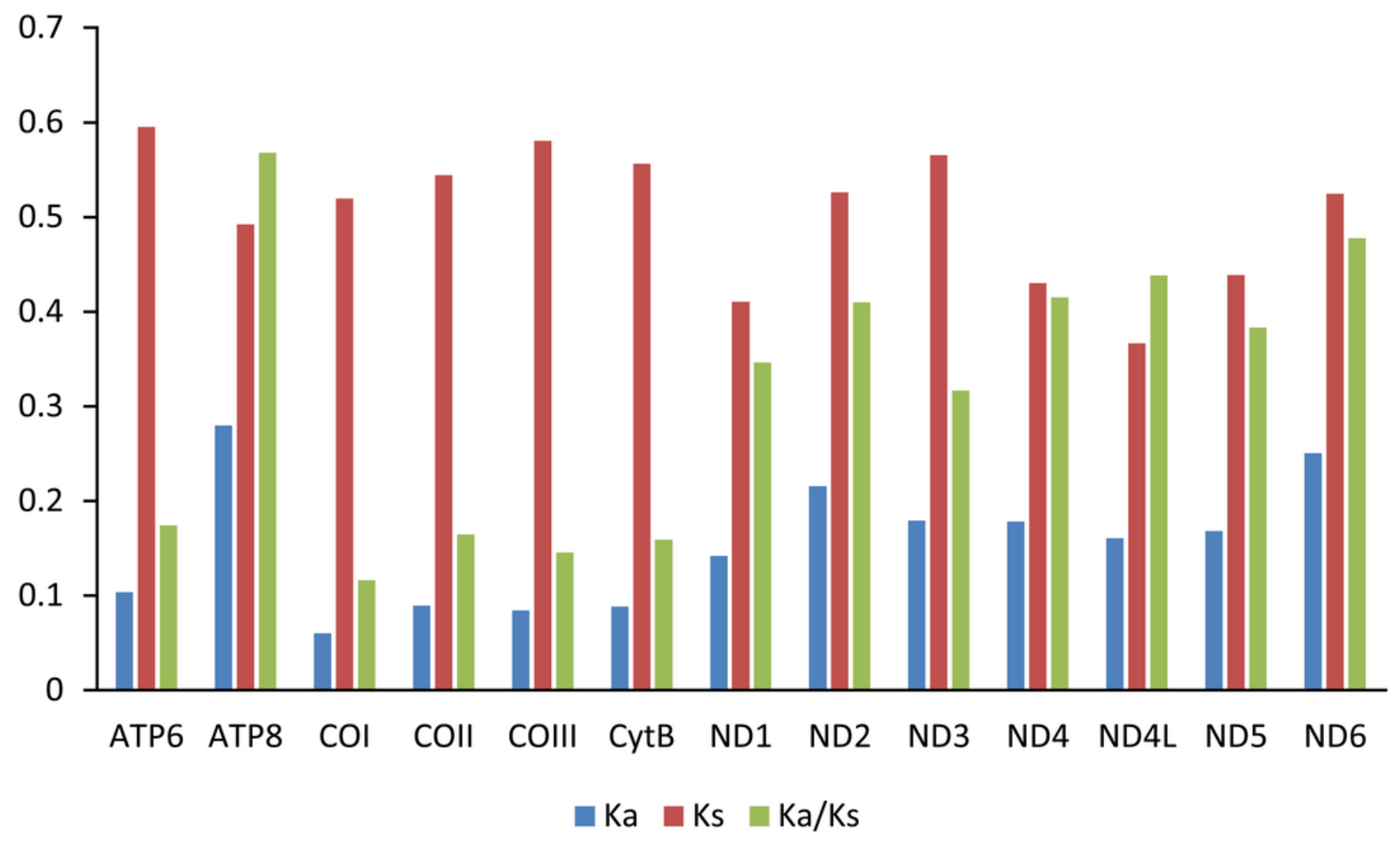


Figure 5

Phylogenetic relationships of six subfamilies within Chironomidae inferred from mitogenomes.

(a) Topology obtained based on PCG123; (b) Topology obtained based on PCG123R; (c) Topology obtained based on PCG12; (d) Topology obtained based on PCG12R; (e) Topology obtained based on AA. Numbers at the nodes are BI posterior probabilities (left) and ML bootstrap values (right).

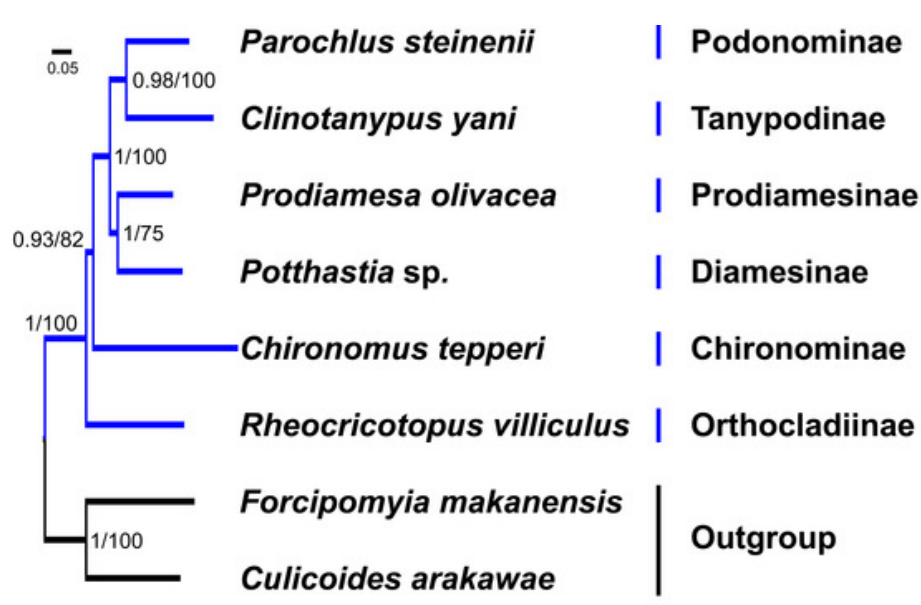

a

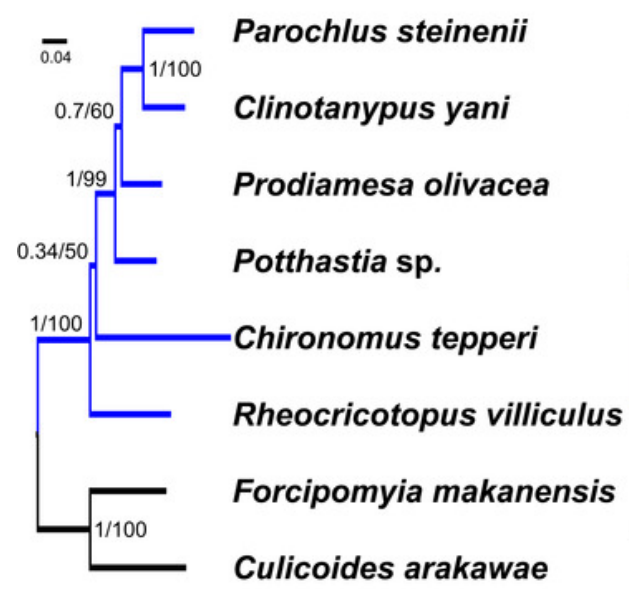

C

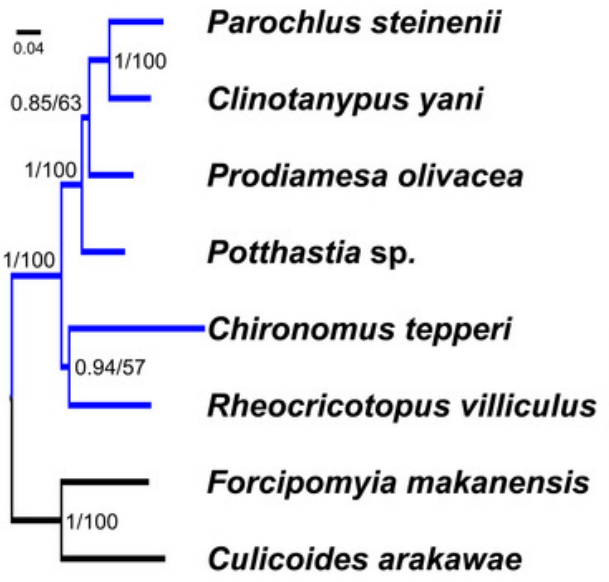

d

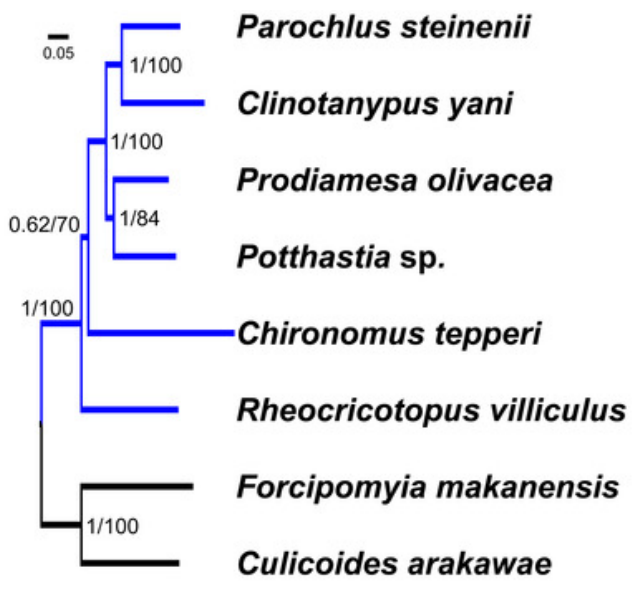

b

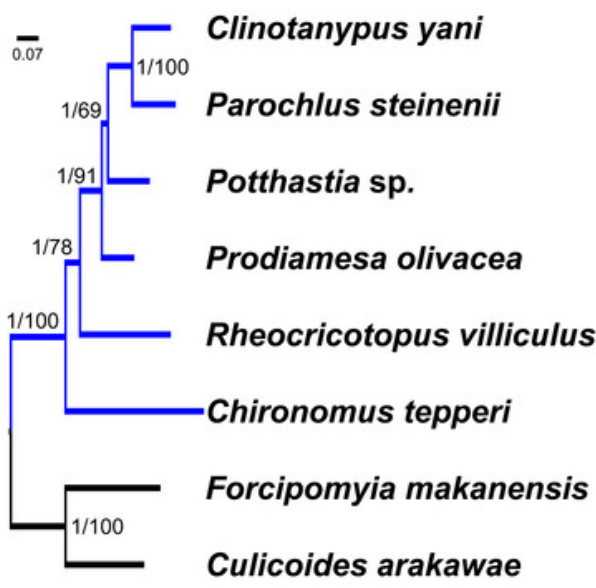

$\mathbf{e}$ 


\section{Table $\mathbf{1}$ (on next page)}

Taxonomic information, sampling metadata, GenBank accession numbers and references of mitochondrial genomes used in the study. 
1 Table 1. Taxonomic information, sampling metadata, GenBank accession numbers and references of mitochondrial genomes

2 used in the study.

\begin{tabular}{|c|c|c|c|c|c|c|}
\hline Family & Subfamily & Species & Life stage & Sampling metadata & $\begin{array}{l}\text { Accession } \\
\text { no }\end{array}$ & Reference \\
\hline Ceratopogonidae & Ceratopogoninae & $\begin{array}{l}\text { Culicoides } \\
\text { arakawae }\end{array}$ & & & NC_009809 & $\begin{array}{l}\text { Matsumoto } \\
\text { et al. (2009) }\end{array}$ \\
\hline Ceratopogonidae & Forcipomyiinae & $\begin{array}{l}\text { Forcipomyia } \\
\text { makanensis }\end{array}$ & & Makan, Zunyi, Guizhou, China, $27.630765^{\circ} \mathrm{N}, 106.848949^{\circ} \mathrm{E}$ & MK000395 & $\begin{array}{l}\text { Jiang et al. } \\
(2019)\end{array}$ \\
\hline Chironomidae & Chironominae & $\begin{array}{l}\text { Chironomus } \\
\text { tepperi }\end{array}$ & & & JN861749 & $\begin{array}{l}\text { Beckenbach } \\
(2012)\end{array}$ \\
\hline Chironomidae & Diamesinae & Potthastia sp. & Adult male & $\begin{array}{l}\text { Wuying, Yichun, Heilongjiang, China, } 48.0869^{\circ} \mathrm{N}, 129.2470^{\circ} \mathrm{E}, 27-\mathrm{Jui}- \\
\text { 2016, leg. C. Song }\end{array}$ & MW373523 & This study \\
\hline Chironomidae & Orthocladiinae & $\begin{array}{l}\text { Rheocricotopus } \\
\text { villiculus }\end{array}$ & Adult male & $\begin{array}{l}\text { Tianmu Mountain National Nature Reserve, Hangzhou, Zhejiang, China, } \\
30.3222^{\circ} \mathrm{N}, 119.442^{\circ} \text { E, 22-Jul-2019, leg. X.-L. Lin }\end{array}$ & MW373526 & This study \\
\hline Chironomidae & Podonominae & $\begin{array}{l}\text { Parochlus } \\
\text { steinenii }\end{array}$ & & $\begin{array}{l}\text { King George Island, West, Antarctica, } 62.2333^{\circ} \mathrm{S}, 58.7833^{\circ} \mathrm{W} \text {, summer in } \\
2015\end{array}$ & KT003702 & $\begin{array}{l}\text { Kim et al. } \\
(2016)\end{array}$ \\
\hline Chironomidae & Prodiamesinae & $\begin{array}{l}\text { Prodiamesa } \\
\text { olivacea }\end{array}$ & Larva & $\begin{array}{l}\text { Jiuzhaigou Valley Scenic and Historic Interest Area, Sichuan, China, } \\
33.1928^{\circ} \mathrm{N}, 103.8942^{\circ} \mathrm{E}, 12-\mathrm{Jul}-2019 \text {, leg. X.-Y. Ge }\end{array}$ & MW373525 & This study \\
\hline Chironomidae & Tanypodinae & $\begin{array}{l}\text { Clinotanypus } \\
\text { yani }\end{array}$ & Adult male & $\begin{array}{l}\text { Jiulongshan Nature Reserve, Guangyuan, Sichuan, China, } 31.976379^{\circ} \mathrm{N} \text {, } \\
106.035644^{\circ} \mathrm{E}, 8 \text {-Aug-2017, leg. C. Song }\end{array}$ & MW373524 & This study \\
\hline
\end{tabular}

3 


\section{Table 2 (on next page)}

Nucleotide composition of mitochondrial genomes of the six Chironomidae species. 
1 Table 2. Nucleotide composition of mitochondrial genomes of the six Chironomidae species.

\begin{tabular}{|c|c|c|c|c|c|c|c|c|c|c|}
\hline & Species & $\begin{array}{c}\text { Whole } \\
\text { genome }\end{array}$ & $\begin{array}{c}\text { Protein coding } \\
\text { genes }\end{array}$ & $\begin{array}{c}\text { First codon } \\
\text { position } \\
\end{array}$ & $\begin{array}{l}\text { Second codon } \\
\text { position }\end{array}$ & $\begin{array}{c}\text { Third codon } \\
\text { position }\end{array}$ & $\begin{array}{l}\text { tRNA } \\
\text { genes }\end{array}$ & $\begin{array}{c}12 \mathrm{~S} \\
\text { rRNA } \\
\end{array}$ & $\begin{array}{c}16 \mathrm{~S} \\
\text { rRNA }\end{array}$ & $\begin{array}{c}\text { Control } \\
\text { region }\end{array}$ \\
\hline \multirow{6}{*}{$\mathrm{A}+\mathrm{T} \%$} & Chironomus tepperi & 76.7 & 74.3 & 67.6 & 67.6 & 87.6 & 79.0 & 82.6 & 84.3 & 93.0 \\
\hline & Potthastia sp. & 76.8 & 74.7 & 69.0 & 66.1 & 88.9 & 76.8 & 78.1 & 82.7 & 93.3 \\
\hline & $\begin{array}{l}\text { Rheocricotopus } \\
\text { villiculus }\end{array}$ & 77.3 & 74.4 & 69.6 & 67.4 & 86.0 & 79.5 & 84.1 & 84.4 & 93.7 \\
\hline & Parochlus steinenii & 72.4 & 69.0 & 64.6 & 64.7 & 77.5 & 73.2 & 76.4 & 80.1 & 85.5 \\
\hline & $\begin{array}{l}\text { Prodiamesa } \\
\text { olivacea }\end{array}$ & 75.8 & 73.4 & 66.7 & 65.5 & 88.2 & 76.2 & 78.1 & 81.9 & 89.2 \\
\hline & Clinotanypus yani & 75.0 & 72.5 & 65.4 & 65.1 & 87.0 & 75.7 & 79.1 & 81.3 & 88.7 \\
\hline \multirow{6}{*}{$\begin{array}{l}\text { AT- } \\
\text { Skew }\end{array}$} & Chironomus tepperi & -0.14 & -0.20 & -0.09 & -0.41 & -0.13 & 0.03 & -0.01 & 0.00 & -0.11 \\
\hline & Potthastia sp. & -0.13 & -0.20 & -0.10 & -0.39 & -0.12 & 0.03 & 0.01 & 0.04 & -0.05 \\
\hline & $\begin{array}{l}\text { Rheocricotopus } \\
\text { villiculus }\end{array}$ & -0.12 & -0.18 & -0.09 & -0.40 & -0.08 & 0.02 & 0.01 & 0.05 & -0.07 \\
\hline & Parochlus steinenii & -0.11 & -0.19 & -0.09 & -0.40 & -0.11 & 0.04 & 0.05 & 0.03 & 0.06 \\
\hline & $\begin{array}{l}\text { Prodiamesa } \\
\text { olivacea }\end{array}$ & -0.12 & -0.19 & -0.10 & -0.40 & -0.09 & 0.03 & 0.04 & 0.01 & 0.02 \\
\hline & Clinotanypus yani & -0.13 & -0.19 & -0.10 & -0.39 & -0.10 & 0.02 & 0.00 & 0.03 & -0.08 \\
\hline \multirow{6}{*}{$\begin{array}{l}\text { GC- } \\
\text { Skew }\end{array}$} & Chironomus tepperi & -0.06 & -0.02 & 0.19 & -0.18 & -0.12 & -0.13 & -0.37 & -0.36 & -0.43 \\
\hline & Potthastia sp. & -0.03 & 0.02 & 0.27 & -0.16 & -0.15 & -0.12 & -0.24 & -0.28 & -0.31 \\
\hline & $\begin{array}{l}\text { Rheocricotopus } \\
\text { villiculus }\end{array}$ & -0.04 & -0.01 & 0.25 & -0.18 & -0.17 & -0.09 & -0.23 & -0.34 & -0.19 \\
\hline & Parochlus steinenii & -0.04 & -0.01 & 0.21 & -0.17 & -0.10 & -0.06 & -0.21 & -0.26 & -0.18 \\
\hline & $\begin{array}{l}\text { Prodiamesa } \\
\text { olivacea }\end{array}$ & -0.04 & 0.00 & 0.25 & -0.16 & -0.21 & -0.09 & -0.24 & -0.29 & -0.16 \\
\hline & Clinotanypus yani & -0.06 & 0.00 & 0.24 & -0.18 & -0.18 & -0.12 & -0.28 & -0.34 & -0.39 \\
\hline
\end{tabular}

2 